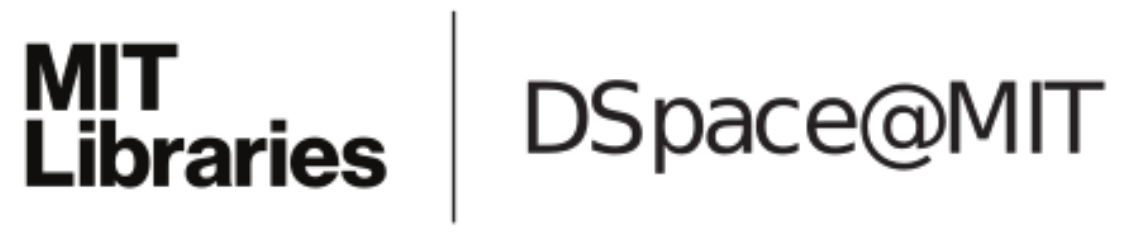

\author{
MIT Open Access Articles
}

Controlling chemical oscillations in heterogeneous Belousov-Zhabotinsky gels via mechanical strain

The MIT Faculty has made this article openly available. Please share how this access benefits you. Your story matters.

Citation: Yashin, Victor V., Krystyn J. Van Vliet, and Anna C. Balazs. “Controlling chemical oscillations in heterogeneous Belousov-Zhabotinsky gels via mechanical strain." Physical Review E 79.4 (2009): 046214. (C) 2010 The American Physical Society.

As Published: http://dx.doi.org/10.1103/PhysRevE.79.046214

Publisher: American Physical Society

Persistent URL: http://hdl.handle.net/1721.1/51008

Version: Final published version: final published article, as it appeared in a journal, conference proceedings, or other formally published context

Terms of Use: Article is made available in accordance with the publisher's policy and may be subject to US copyright law. Please refer to the publisher's site for terms of use. 


\title{
Controlling chemical oscillations in heterogeneous Belousov-Zhabotinsky gels via mechanical strain
}

\author{
Victor V. Yashin, ${ }^{1}$ Krystyn J. Van Vliet, ${ }^{2}$ and Anna C. Balazs ${ }^{1, *}$ \\ ${ }^{1}$ Department of Chemical Engineering, University of Pittsburgh, Pittsburgh, Pennsylvania 15261, USA \\ ${ }^{2}$ Department of Materials Science and Engineering, Massachusetts Institute of Technology, Cambridge, Massachusetts 02139, USA
}

(Received 23 October 2008; published 22 April 2009)

\begin{abstract}
We performed theoretical and computational studies to determine the effect of an applied mechanical strain on the dynamic behavior of heterogeneous polymer gels undergoing the oscillatory Belousov-Zhabotinsky (BZ) reaction. In these spatially heterogeneous gels, the catalyst for the reaction is localized in specific patches within the polymer network and the BZ reaction only occurs within these catalyst-containing patches, which we refer to as BZ patches. We focused on a model for a one-dimensional system, and further assumed that the BZ reaction did not affect the degree of swelling within the gel. For gels having one and two BZ patches, we found that a tensile or compressive strain could induce transitions between the oscillatory and nonoscillatory, steady-state regimes of the system. For certain values of the BZ stoichiometric parameter $f$, these transitions could exhibit a hysteresis. In systems having two oscillating BZ patches, an applied strain could cause a switching between the in-phase and out-of-phase synchronization of the oscillations. The ability to controllably alter the dynamic behavior of BZ gels through mechanical deformations opens up the possibility of using these materials in the design of chemo-mechanical sensors.
\end{abstract}

DOI: 10.1103/PhysRevE.79.046214

PACS number(s): 82.40.Bj, 82.40.Ck, 47.54.-r

\section{INTRODUCTION}

Mechanochemistry involves the activation of chemical reactivity by an external mechanical deformation or force. The field of mechanochemistry is arguably less developed than thermo-, photo- or electro-chemistry [1]. In particular, the control of chemical reactivity through an applied mechanical strain remains a relatively unexplored and potentially rich area of science. Understanding how mechanical strain produces chemical changes in materials could, for example, present opportunities for creating stimuli-responsive systems.

Using theory and simulation, we recently have begun to probe the effects of mechanical deformation on a particular class of responsive materials, namely, polymer gels undergoing the Belousov-Zhabotinsky (BZ) reaction [2]. BZ gels are unique because the polymer network can expand and contract periodically in the absence of applied external stimuli $[3,4]$. This autonomous, self-oscillatory swelling behavior is due to a ruthenium catalyst, which participates in the BZ reaction and is covalently bonded to the polymers $[3,4]$. The $\mathrm{BZ}$ reaction generates a periodic oxidation and reduction in the anchored metal ion, and these chemical oscillations induce the rhythmic swelling and deswelling in the gel.

While the BZ gels can pulsate without external controls, we have found that mechanical pressure can be harnessed to manipulate the oscillatory behavior of these gels [2]. In order to probe these effects, we developed computational models to simulate the behavior of BZ gels in two dimensions $[5,6]$ and more recently, in three dimensions (3D) [7]. [The twodimensional (2D) model effectively captures the behavior of gels that are confined between two surfaces, i.e., the gels are allowed to oscillate in the lateral directions, but the height of

\footnotetext{
*balazs@pitt.edu
}

the sample is kept fixed.] Using these models, we isolated systems where the applied pressure induced chemical oscillations in an initially nonoscillatory system [2]. With the aid of the 2D model, we also pinpointed a scenario where a compression induces both oscillations and the autonomous rotation of the entire BZ gel sample [2].

In the above studies, the BZ gels were assumed to be homogeneous, i.e., the catalyst was uniformly distributed throughout the sample. In addition to such homogeneous BZ gels, we have modeled the behavior of heterogeneous or chemically patterned gels, where the catalyst was confined to distinct patches within the polymer network [8]. In such systems, the chemical oscillations only occur within these BZ patches. Our studies showed that the oscillations in neighboring patches can become synchronized, with these regions pulsating in phase or out of phase with respect to each other [8].

These heterogeneous systems are of interest because the patterning of the gel provides a route for designing systems with well-defined dynamical behavior. For example, our 2D simulations of heterogeneous gels revealed that the lateral separation, $\Delta x$, between two BZ patches controls the synchronization dynamics of these patches. By varying $\Delta x$, we produced a switching between the in-phase and out-of-phase modes of synchronization in heterogeneous gels containing a linear array of BZ patches [8].

The lateral separation between patches in a sample of the heterogeneous BZ gel would be fixed during the fabrication of the gel. After fabrication, however, the distance could be changed by applied mechanical deformation of the gel or by induced swelling/shrinkage of the polymer network. The resulting mechanical strain could potentially drive a system into the oscillatory state or alter the synchronization between multiple oscillating patches. If this form of mechanochemistry was shown to occur, the heterogeneous BZ gels could be utilized as a functional material that alters its functionality in a response to an external mechanical action. 
In this paper, we use theory and simulation to investigate the effects of deforming self-oscillating polymer gels that contain spatially heterogeneous distributions of BZ catalyst. To facilitate the theoretical analysis, we consider a onedimensional (1D) model of the heterogeneous BZ gel. This 1D model describes gels that are confined within a narrow tube, or capillary, and is an approximate description of tensile or compressive strain in 2D gels comprising arrays of high-aspect-ratio BZ patches.

In the $1 \mathrm{D}$ model, the mechanical strain is introduced by increasing (or decreasing) the length of the sample, effectively simulating an applied tensile (or compressive) strain on the gel. A further simplification is achieved by assuming that the ongoing $\mathrm{BZ}$ reaction does not affect the degree of swelling within the gel. In other words, we assume that the chemical oscillations in the reaction do not produce mechanical oscillations of the gel; we refer to these materials as nonresponsive gels. In fact, such nonresponsive gels have been fabricated $[9,10]$. On the other hand, deformations of the gel affect the BZ reaction through variations in the volume fraction of polymer within the sample.

We start by considering a system that contains a single BZ patch and isolate the conditions that drive the system into the oscillatory regime of the BZ reaction. The results of this study allow us to pinpoint the model parameters for which the oscillations can be switched "on" and "off" by changing the length of the system, e.g., by inducing a mechanical strain. Then, we consider a polymer gel that has two catalystcontaining patches. We focus on the existence, coexistence, and stability of the in-phase and out-of-phase oscillations in a 1D system subject to mechanical strain and determine how the system parameters affect the dynamical behavior.

\section{METHODOLOGY}

Our system consists of a swollen polymer gel; within this gel, the polymer-tethered BZ catalysts are localized in specific patches (BZ patches) that are arranged along the $X$ axis. The material is treated as a purely one-dimensional system; this assumption is reasonable if the patterned gel is confined in a thin capillary tube, or a 2D film of gel contains an array of long, parallel strips of the BZ patches. The cross-link density and the volume fraction of polymer are assumed to be spatially uniform throughout the whole sample. In the nondeformed state, the gel sample in characterized by the polymer volume fraction $\phi_{0}$ and the initial length $L_{0}$. After this sample is placed in a capillary tube (oriented along the $X$ axis), it becomes swollen so that its degree of swelling in the longitudinal direction is $\lambda$ and the degree of swelling in the transverse direction is $\lambda_{\perp}$. The polymer volume fraction $\phi$ and the gel length $L$ in the swollen state are

$$
\phi=\phi_{0} \lambda^{-1} \lambda_{\perp}^{-2}
$$

and $L=\lambda L_{0}$, respectively.

We assume that the $\mathrm{BZ}$ gel is nonresponsive, i.e., the $\mathrm{BZ}$ reaction does not affect the polymer-solvent interactions. The behavior of this system is governed by reaction-diffusion equations that describe the $\mathrm{BZ}$ reaction both inside and outside the catalyst-containing patches. To capture this behavior, we utilize the Oregonator model [11], which describes the $\mathrm{BZ}$ reaction in terms of the concentrations of the diffusing reactant, $u$ and the oxidized catalyst, $v$. Within a patch, the equations in the dimensionless form are

$$
\begin{gathered}
\partial_{t} u=\partial_{x x} u+F(u, v, \phi), \\
\partial_{t} v=\varepsilon G(u, v, \phi) .
\end{gathered}
$$

The original Oregonator model, however, was formulated for the $\mathrm{BZ}$ reaction in solution (i.e., not in a gel) and the reaction rate functions $F$ and $G$ just depended on the concentrations $u$ and $v$ [11]. We recently modified this model to account for the presence of the polymer network under the assumption that the polymer acts like a neutral diluent $[6,12]$. Now, both $F$ and $G$ also depend on the volume fraction of polymer, $\phi$. Thus, for the BZ gels, we utilize the following equations $[6,12]$ :

$$
\begin{gathered}
F(u, v, \phi)=(1-\phi)^{2} u-u^{2}-(1-\phi) f v \frac{u-q(1-\phi)^{2}}{u+q(1-\phi)^{2}}, \\
G(u, v, \phi)=(1-\phi)^{2} u-(1-\phi) v .
\end{gathered}
$$

The stoichiometric parameter $f$ and the dimensionless parameters $\varepsilon$ and $q$ have the same meaning as in the original Oregonator [11]. The parameter $\varepsilon$ in Eq. (3) is a ratio of two reaction rates; one reaction rate is for the reduction of the metal-ion catalyst during the oxidation of the organic substrate and the other is for the oxidation of the catalyst mediated by the $\mathrm{BZ}$ reactant $u$ [11]. Hence, $\varepsilon$ depends on both the composition of the reaction substrate and temperature. The parameter $q$ in Eq. (5) is a combination of reaction rate constants and thus, depends only on temperature [11].

Since the reactant $u$ is a product of the reaction, $u$ simply diffuses and decays outside of the patches; thus, within these catalyst-free regions, the evolution of $u$ is given by [13]

$$
\partial_{t} u=\partial_{x x} u-u^{2} .
$$

The concentration $u$ and its spatial derivative $\partial_{x} u$ are continuous at the boundaries between the catalyst-containing and catalyst-free regions of the gel.

As is evident from Eqs. (2)-(5), the local volume fraction of polymer $\phi$ affects the kinetics of the BZ reaction. In general, Eqs. (2) and (3) must be supplemented by an equation that describes the spatiotemporal evolution of $\phi$. The theoretical analysis can be significantly simplified if the polymer gel is assumed to be in its swollen equilibrium state at the given boundary conditions. In this case, $\phi=$ const since we are considering a nonresponsive gel with a spatially uniform cross-link density. Then, the $\phi$ dependence can be effectively eliminated from Eqs. (4) and (5) through the following rescaling: $u(1-\phi)^{-2} \rightarrow u, v(1-\phi)^{-3} \rightarrow v$, and $t(1-\phi)^{2} \rightarrow t[6]$. If, in addition, we change the length scale as $\lambda^{-1} x \rightarrow x$, then the reaction-diffusion equations within the patches, Eqs. (2) and (3), take the following form:

$$
\partial_{t} u=D_{\lambda} \partial_{x x} u+F(u, v),
$$




$$
\partial_{t} v=\varepsilon_{\lambda} G(u, v) .
$$

Here

$$
D_{\lambda}=\lambda^{-2}(1-\phi)^{-2}, \quad \varepsilon_{\lambda}=\varepsilon(1-\phi)^{-1}
$$

and the rate functions $F$ and $G$ are given by the original Oregonator equations of Tyson and Fife [11]:

$$
\begin{gathered}
F(u, v)=u(1-u)-f v \frac{u-q}{u+q}, \\
G(u, v)=u-v .
\end{gathered}
$$

The evolution of $u$ in the catalyst-free regions [Eq. (6)] takes the following form after the rescaling:

$$
\partial_{t} u=D_{\lambda} \partial_{x x} u-u^{2} .
$$

Thus, after the rescaling, the problem is solved on the spatial interval of length $L_{0}$; the values of the polymer volume fraction $\phi$ and longitudinal degree of swelling $\lambda$ affect the system behavior only through the effective diffusion coefficient $D_{\lambda}$ and parameter $\varepsilon_{\lambda}$ given by Eq. (9). It is worth recalling that the polymer volume fraction $\phi$ itself depends on $\lambda$ through Eq. (1).

Note that $\lambda$ is the longitudinal degree of stretching, which can be changed either by applying mechanical force to the ends of the gel or altering the external conditions that affect the swelling of the gel. In this paper, we do not specify the particular action that changes $\lambda$. We focus on how the longitudinal stretching $\lambda$ affects the dynamics of the heterogeneous gel provided that the gel size in the transverse direction remains unchanged $\left(\lambda_{\perp}=\right.$ const). To achieve this goal, we first obtain the steady-state solution of the model, and then perform a linear stability analysis of this solution. As both the steady state and its stability to small perturbations are affected by the value of $\lambda$, we find as a result how the regions of the oscillatory solutions and the in- and out-ofphase synchronization modes depend on the longitudinal stretching.

\section{A. Steady-state solutions}

In the heterogeneous gel, the steady-state distributions of the concentrations $u$ and $v$ within the patches are described by the equations

$$
D_{\lambda} u^{\prime \prime}+F(u, v)=0, \quad G(u, v)=0,
$$

where the rate functions $F$ and $G$ are given by Eqs. (10) and (11), respectively. For simplicity, in the ensuing discussion, we will refer to the catalyst-free gel as the "neutral" gel. In the neutral gel between the patches, the concentration $u$ obeys the equation

$$
D_{\lambda} u^{\prime \prime}-u^{2}=0 \text {. }
$$

We use the notation $u^{\prime}=d u / d x$. As stated above, the function $u(x)$ and its first derivative are continuous at the boundaries between the BZ and neutral gels. We note that $v(x)=u(x)$ in the steady state due to the particular form of the function $G(u, v)$, Eq. (11). The steady-state equation for $u$ can be written as

$$
D_{\lambda} u^{\prime \prime}=-\frac{d U_{r}}{d u}
$$

where the subscript $r=p, g$ denotes the regions within the catalyst-containing BZ patches (" $p$ ") and the neutral gel (" $g$ "). It is worth noting that Eq. (15) is mathematically equivalent to the equation of motion of a material point of mass $D_{\lambda}$ in the potential field $U_{r}(u)$. The potential is different in the patch and gel areas:

$$
U_{p}(u)=1 / 2 u^{2}(1-f-2 / 3 u)+2 f q[u-q \ln (u+q)],
$$

$$
U_{g}(u)=-1 / 3 u^{3} .
$$

We enumerate alternating sequence of the catalystcontaining and neutral gel intervals along the sample by a set of successive integer numbers $n$, so that the even and odd numbers correspond to the $\mathrm{BZ}$ patches and neutral gel areas, respectively. The $n$th area of the heterogeneous gel occupies the interval $x \in\left[x_{n}, x_{n+1}\right]$ on the $X$ axis, and has a length $L_{n}$ $=x_{n+1}-x_{n}$. Correspondingly, the steady-state solution within the interval $n$ is denoted as $u_{n}(x)$, and the following notation for the potential functions is used:

$$
U_{n}(u)= \begin{cases}U_{p}(u), & \text { even } n \\ U_{g}(u), & \text { odd } n .\end{cases}
$$

Equation (15) can be integrated within each interval to obtain an analog of the energy conservation law:

$$
\frac{D_{\lambda}\left(u^{\prime}\right)^{2}}{2}+U_{n}(u)=E_{n},
$$

where $E_{n}$ is the integration constant (energy) corresponding to the interval $n$. Thus, the problem of finding the steadystate solutions is reduced to solving a system of first-order ODE's, Eq. (19). Equation (19) gives the following implicit solution for the function $u_{n}(x)$ :

$$
\pm\left(2 D_{\lambda}\right)^{-1 / 2} \int\left[E_{n}-U_{n}(u)\right]^{-1 / 2} d u=x+C_{n} .
$$

Here, $C_{n}$ is the integration constant. The sign on the left-hand side of Eq. (20) coincides with the sign of the derivative $u^{\prime}(x)$. To determine the integration constants $E_{n}$ and $C_{n}$, the boundary conditions must be fixed at the left and right ends of the gel, and the interval solutions $u_{n}(x)$ should be matched at the boundaries between the intervals:

$$
u_{n-1}\left(x_{n}\right)=u_{n}\left(x_{n}\right), \quad u_{n-1}^{\prime}\left(x_{n}\right)=u_{n}^{\prime}\left(x_{n}\right) .
$$

We will also use the notation $u_{n}=u_{n}\left(x_{n}\right)$ and

$$
Q_{r}\left(u_{a}, u_{b}, E\right)=\left(2 D_{\lambda}\right)^{-1 / 2} \int_{u_{a}}^{u_{b}}\left[E-U_{r}(u)\right]^{-1 / 2} d u, \quad r=p, g .
$$

Equations (18) and (19) describe all possible steady-state solutions within the catalyst-containing and neutral gel areas. It is instructive to represent the solutions graphically in terms of phase portraits, i.e., by plotting the "velocity" $u$ ' as a 
(a)

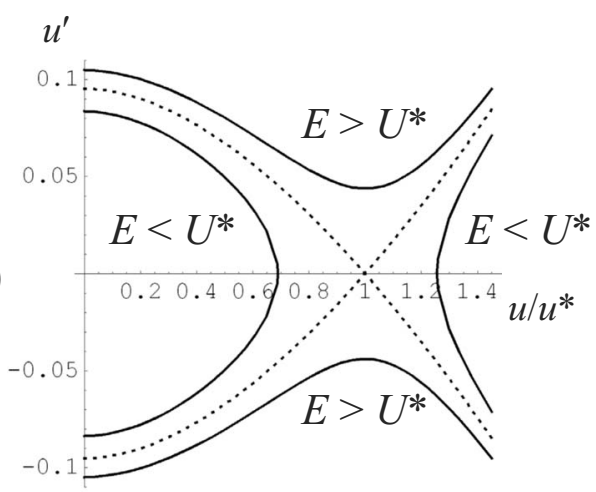

(b)

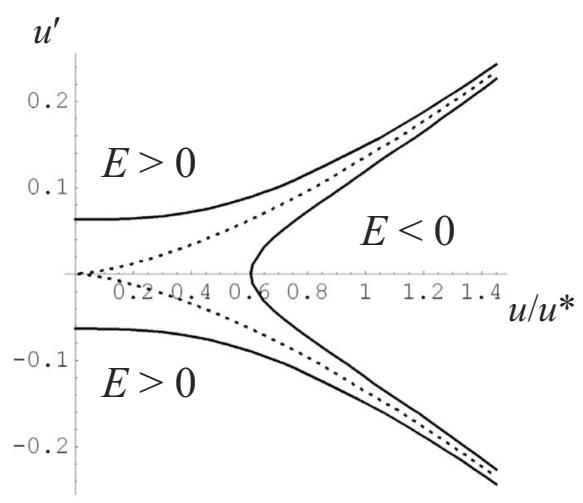

(c)

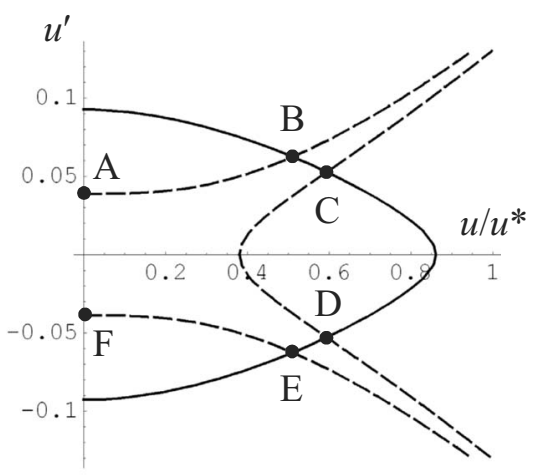

FIG. 1. Phase portraits of Eq. (19) within (a) BZ patch, (b) neutral gel, and (c) symmetric heterogeneous gel.

function of the "coordinate" $u$ at various values of the "energy" E. Figures 1(a) and 1(b) show the phase portraits of Eqs. (18) and (19) for the BZ patch and neutral gel, respectively. As seen in Fig. 1, the structure of a steady-state solution qualitatively depends on the value of $E$. In Fig. 1(a), there is the separatrix curve indicated by the dotted line, which corresponds to $E=U_{p}\left(u^{*}\right)$, where $u^{*}$ is the steady-state concentration of $u$ in the homogeneous system obtained from Eq. (13) at $u^{\prime \prime}=0$ :

$$
u^{*}=\frac{1}{2}\left[1-f-q+\sqrt{(1-f-q)^{2}+4 q+8 f q}\right] .
$$

In Fig. 1(b), the separatrix corresponds to $E=0$.

The steady-state distribution of the diffusing reactant $u$ in a heterogeneous gel can be obtained by a proper combination (a)

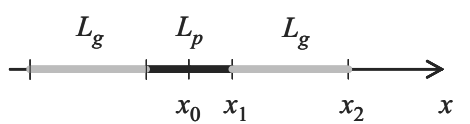

(b)

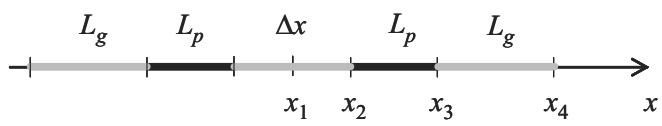

FIG. 2. Coordinate notations for the symmetric gel containing (a) one patch and (b) two patches. The BZ patches are marked in black.

of the trajectories shown in Figs. 1(a) and 1(b). Let us consider the symmetric heterogeneous gels having one and two patches as shown in Fig. 2. We assume that $u=0$ at the ends of the sample. Then, the steady-state solutions can be found as schematically shown in Fig. 1(c). In this figure, the solid line indicates the steady-state solution within the BZ patches, and the dashed lines correspond to those in the neutral gel areas. The trajectory ABEF gives the solution in the symmetric heterogeneous gel having one patch. In the case of two patches, the solution is given by the trajectory ABDCEF.

Let us now find the steady-state solutions in the explicit form. As indicated in Fig. 2, the length of the catalystcontaining patches is $L_{p}$, and the distance from the patches to the sample ends is $L_{g}$. The lateral separation between patches, or interpatch distance, is $\Delta x$ [see Fig. 2(b)]. We seek the spatially symmetric solution, so it is sufficient to consider only one half of the gel sample, i.e., the areas $x_{0} \leq x \leq x_{2}$ in Fig. 1(a) and $x_{1} \leq x \leq x_{4}$ in Fig. 2(b). We note that the numbering of the interval boundaries in Fig. 2 corresponds to the adopted notation for the catalyst-containing and neutral gel areas.

In the gel having one patch (see Fig. 1), the distribution of $u$ exhibits a maximum at the center of the BZ patch [see Fig. $1(\mathrm{c})]$, so that $u_{0}^{\prime}\left(x_{0}\right)=0$ and $u_{0}>u_{1}$. It also follows from Eqs. (18) and (19) that $E_{0}=U_{p}\left(u_{0}\right)$ at $u_{0}^{\prime}\left(x_{0}\right)=0$. As a result, Eq. (20) applied to the BZ patch takes the following form:

$$
Q_{p}\left[u_{1}, u_{0}, U_{p}\left(u_{0}\right)\right]=1 / 2 L_{p} .
$$

Within the neutral gel interval, $u_{2}=0$ due to the chosen boundary conditions, so that $E_{1} \geq 0$ and

$$
Q_{g}\left(0, u_{1}, E_{1}\right)=L_{g} \text {. }
$$

The interval solutions are matched at the boundary between them through Eqs. (19) and (21) giving

$$
U_{p}\left(u_{0}\right)-U_{p}\left(u_{1}\right)=E_{1}-U_{g}\left(u_{1}\right) .
$$

Equations (24)-(26) are solved numerically to obtain the unknown values of $u_{0}, u_{1}$, and $E_{1}$. Finally, the steady-state distribution of $u$ is found explicitly by solving Eq. (19) via numerical methods. It is worth noting that if the BZ patch is placed within an infinite system, i.e., if $L_{g} \rightarrow \infty$, then $E_{1}=0$ and the distribution of $u$ within the neutral gel can be found analytically to be

$$
u_{1}^{-1 / 2}(x)=u_{1}^{-1 / 2}+\left(6 D_{\lambda}\right)^{-1 / 2}\left(x-x_{1} / 2\right) .
$$

In this limiting case, the values of $u_{0}$ and $u_{1}$ are found by solving Eqs. (24) and (26). We also note that the maximum 
value of $u$ within a BZ patch of finite length $L_{p}$ is always smaller than the steady-state value in the infinite system; $u_{0}$ approaches $u^{*}$ as $L_{p} \rightarrow \infty$. It can be shown that the integral on the left-hand side of Eq. (24) is a monotonously increasing function of $u_{0}$ that diverges as $u_{0}$ approaches $u^{*}$ from below.

The steady-state solution for the gel having two BZ patches can be found by taking the same steps as described above. The profile of $u$ goes through a minimum in the middle of the neutral gel area separating the two patches [see Fig. 1(c)], so that $u_{2}>u_{1}$, and $E_{1}=U_{g}\left(u_{1}\right)$. Therefore, Eq. (20) for this interval can be written as

$$
Q_{g}\left[u_{1}, u_{2}, U_{g}\left(u_{1}\right)\right]=1 / 2 \Delta x .
$$

Within the catalyst-containing patch, the concentration $u$ exhibits a maximum with the value $u_{\max }$. Then, $E_{2}=U_{p}\left(u_{\max }\right)$ and

$$
Q_{p}\left[u_{2}, u_{\max }, U_{p}\left(u_{\max }\right)\right]+Q_{p}\left[u_{3}, u_{\max }, U_{p}\left(u_{\max }\right)\right]=L_{p} .
$$

For the neutral gel area at the end of the sample, Eq. (20) takes the form of Eq. (25), namely,

$$
Q_{g}\left(0, u_{3}, E_{3}\right)=L_{g}
$$

Finally, the matching conditions, Eq. (21), at the points $x_{2}$ and $x_{3}$ provide the following respective equations:

$$
\begin{gathered}
U_{g}\left(u_{1}\right)-U_{g}\left(u_{2}\right)=U_{p}\left(u_{\max }\right)-U_{p}\left(u_{2}\right), \\
U_{p}\left(u_{\max }\right)-U_{p}\left(u_{3}\right)=E_{3}-U_{g}\left(u_{3}\right) .
\end{gathered}
$$

Equations (28)-(32) are solved simultaneously to determine the unknown values $u_{1}, u_{2}, u_{3}, u_{\max }$, and $E_{3}$, which are then used to solve Eq. (19) within the corresponding intervals to obtain the steady-state solution $u(x)$.

Thus, we demonstrated that the problem of finding the steady-state solutions is essentially reduced to solving the system of equations [Eqs. (24)-(26) and Eqs. (28)-(32)] for the symmetric heterogeneous gels having one and two catalyst-containing patches, respectively. Solving these equations numerically is complicated by the presence of the integral terms in the left-hand sides of Eq. (24) and Eqs. (28)-(30). We note that the major difficulty is due to the integrals over the BZ patches, $Q_{p}$, in Eqs. (24) and (29). The integrals over the neutral gel intervals, $Q_{g}$, in Eqs. (25), (28), and (30) do not cause major challenges because they can be expressed through hypergeometric functions. A significant simplification can be achieved if the catalyst-containing patches are sufficiently short that the concentration of $u$ does not approach the value of $u^{*}$ [Eq. (23)], which is characteristic for the homogeneous system. In this case, $u(x)$ within the BZ patches can be obtained analytically using a linearization procedure to solve Eq. (13). Within this approximation, Eqs. (24) and (29) are excluded from the systems of equations.

Figure 3 shows the comparison between the steady-state distributions $u(x)$ obtained through the numerical solutions and analytically using the above simplified procedure. It is seen that the results of numerical and analytical calculations are in agreement for the gels having one [Fig. 3(a)] and two
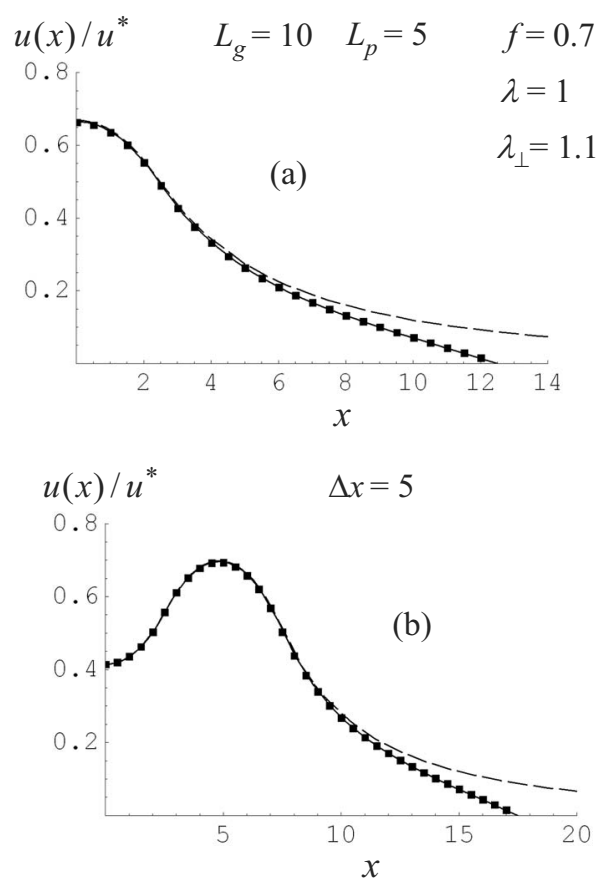

FIG. 3. The steady state in the systems with (a) one and (b) two $\mathrm{BZ}$ patches as obtained by the numerical simulations (symbols) and analytically using the linear approximation within the patches (solid lines). The dashed lines represent the steady states at $L_{g} \rightarrow \infty$.

[Fig. 3(b)] catalyst-containing patches. The dashed lines in Figs. 3(a) and 3(b) represent the steady-state solutions obtained at $L_{g} \rightarrow \infty$, i.e., in the infinite $1 \mathrm{D}$ system. It is seen that the steady-state profiles of $u$ within the patches in the finite gels at $L_{g}=10$ are very close to those in the infinite system.

\section{B. Stability of the steady states}

We study the linear stability of the obtained steady-state solutions in order to reveal the effect of the longitudinal stretching $\lambda$ on the existence of the oscillatory regimes in the heterogeneous BZ gels. For this purpose, Eqs. (6)-(8) are linearized around the spatially nonuniform steady-state distributions $u(x)$ and $v(x)$, and the associated eigenvalue problem is solved. Within the BZ patches, the corresponding equations are

$$
\begin{gathered}
D_{\lambda} \widetilde{u}^{\prime \prime}+F_{u}(u) \tilde{u}+F_{v}(u) \tilde{v}=\Lambda \tilde{u}, \\
\varepsilon_{\lambda}(\tilde{u}-\tilde{v})=\Lambda \tilde{v},
\end{gathered}
$$

where $F_{u}(u)=(\partial / \partial u) F(u, v), F_{v}(u)=(\partial / \partial v) F(u, v)$, and $v=u$. Outside the catalyst-containing patches, the equation is

$$
D_{\lambda} \widetilde{u}^{\prime \prime}-2 u \tilde{u}=\Lambda \tilde{u} \text {. }
$$

The eigenproblem in Eqs. (33)-(35) is solved under the condition that $\tilde{u}=0$ at the ends of the sample. The eigenvalue $\Lambda=\Lambda(\lambda)$ depends on the longitudinal degree of stretching $\lambda$ through the model parameter $\varepsilon_{\lambda}$ and the diffusion coefficient $D_{\lambda}$ in Eqs. (33)-(35). The eigenvalues with $\operatorname{Im} \Lambda \neq 0$ correspond to the oscillatory solutions, which decay or grow in time if $\operatorname{Re} \Lambda<0$ or $\operatorname{Re} \Lambda>0$, respectively. In the latter case, 
the steady-state solution is unstable, meaning that any perturbation develops into the nonlinear oscillations. We are interested in the situations where variations in the stretch $\lambda$ lead to changes in the sign of $\operatorname{Re} \Lambda$ at $\operatorname{Im} \Lambda \neq 0$ (the Hopf bifurcation), i.e., when the dynamics of the system exhibits a qualitative change caused by the mechanical deformation.

\section{Numerical simulations}

The reaction-diffusion equations, Eqs. (2)-(6), were solved numerically using the gel lattice-spring model (gLSM) developed previously by two of us for simulating the dynamics of chemo-responsive BZ gels in two dimensions [6]. This approach utilizes the DVODE routine for solving systems of stiff ordinary differential equations [14]. To solve Eqs. (2)-(6), we applied the 2D gLSM to a stationary lattice that is only two nodes in height, and prohibited the diffusion of the reactant $u$ across the boundaries in the latter direction. With these restrictions, the gLSM becomes an efficient solver for Eqs. (2)-(6), where the spatial derivatives in Eqs. (2) and (6) are approximated by finite differences. The equations for calculating the steady state, Eqs. (28)-(32), and the equations for determining the stability of these steady states, Eqs. (33)-(35), were solved using the MATHEMATICA $^{\mathrm{TM}}$ software.

For the BZ reaction parameters [see Eqs. (8)-(10)], we set $\varepsilon=0.354, q=9.52 \times 10^{-5}$ as in our previous studies [6]. The polymer gel was characterized by $\phi_{0}=0.139$ and $\lambda_{\perp}=1.1$. These parameter values were based, where possible, on available experimental data [6]. The parameters $f$ and $\lambda$ were used as variables. The parameter $f$ was varied in the range $0.6 \leq f \leq 1$. The units of time and length used in our simulations correspond to $T_{0} \sim 1 \mathrm{sec}$ and $L_{0} \sim 40 \mu \mathrm{m}$, respectively [6].The length of the catalyst containing patch $L_{p}$ was taken equal to 3 and $5 L_{0}$. The interpatch spacing $\Delta x$ was varied from 0.5 to $10 L_{0}$. The length of the neutral gel area between the patches and the gels ends $L_{g}$ was set to $10 L_{0}$. Correspondingly, the total respective lengths of the nondeformed system were equal $L_{p}+2 L_{g}$ and $2\left(L_{p}+L_{g}\right)+\Delta x$ in the gels having one and two BZ patches. (When solving the initial equations, Eqs. (2)-(6), in the stretched state, $\Delta x, L_{p}$ and $L_{g}$ are multiplied by the stretch ratio $\lambda$.) The spatial discretization used in the simulations was $1 / 20 L_{0}$. Below, all lengths are measured in the units of $L_{0}$, so it is omitted from the equations for simplicity. For the boundary conditions, we set the concentration of the activator to $u=0$ at the ends of the sample.

\section{RESULTS AND DISCUSSION}

\section{A. Single BZ patch within heterogeneous gel}

The behavior of a heterogeneous gel having one catalystcontaining patch was found to depend upon the length of the patch $L_{p}$. Figure 4(a) shows the steady-state distribution of $u$ in samples with $L_{p}$ equal to 3 and 5 in the case where there is no longitudinal stretching, i.e., $\lambda=1$. It is seen in Fig. 4(a) that the concentration $u$ in both systems is lower than that in the homogeneous gel, $u^{*}$, and that the steady-state values of $u$ are notably lower in the system with the shorter patch.
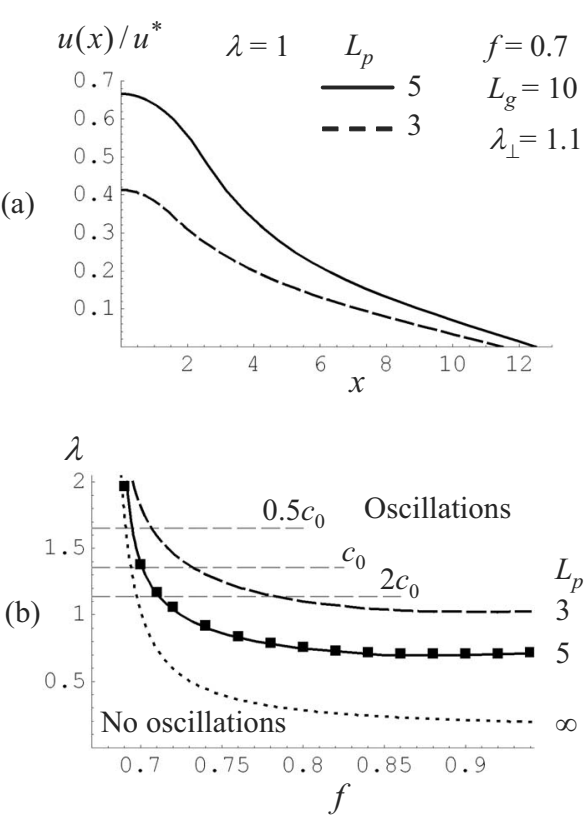

FIG. 4. (a) Steady-state distribution of $u$, and (b) the location of the Hopf bifurcation points as obtained by the linear stability analysis of the steady state in the gel having one patch of length $L_{p}=3$ (dashed line), 5 (solid line), and $\infty$ (dotted line). In (b), the symbols are the data points obtained by the numerical simulations at $L_{p}=5$. Horizontal thin dashed lines in (b) show the equilibrium degree of longitudinal swelling at $20{ }^{\circ} \mathrm{C}$ and the values of cross-link density of $0.5 c_{0}, c_{0}$, and $2 c_{0}$.

The results of the linear stability analysis [see Eqs. (33)-(35)] of these steady states with variations in the degree of stretching $\lambda$ for various values of the stoichiometric parameter $f$ are presented in Fig. 4(b). This figure shows the position of the Hopf bifurcation points on the $\lambda-f$ plane, with the domain of the oscillatory regime of a system being located above the corresponding curve. To obtain the eigenvalues at $L_{p}=3$ and 5, the spatial derivatives in Eqs. (33) and (35) were approximated by the finite differences, with the spatial discretization of $1 / 20$. The resulting matrix eigenproblem was solved numerically using the MATHEMATICA ${ }^{\mathrm{TM}}$ software. Recall that due to the applied rescaling, the stretching $\lambda$ enters Eqs. (33)-(35) only through $D_{\lambda}$ and $\varepsilon_{\lambda}$, so the eigenproblem is solved on the nonstretched intervals of $L_{p}$ and $L_{g}$. It is worth noting that fine spatial discretization is essential for finding the correct location of the bifurcation points at small values of $f$, i.e., in proximity to the left boundary of the oscillatory domain in Fig. 4(b). For example, at $L_{p}=5$, the discretization of $1 / 4$ yields the bifurcation value of $\lambda$ at $f=0.7$ with the relative deviation of about $14 \%$, whereas at $f=0.9$ the relative deviation is about $3 \%$.

To verify the results of the linear stability analysis, we conducted the numerical simulations of the $1 \mathrm{D}$ model at $L_{p}$ $=5$, and the obtained data are shown in Fig. 4(b) by the solid square symbols. The latter data points were obtained by starting the simulations at sufficiently low values of $\lambda$, at which the system exhibits no oscillations. Then, the longitudinal strain $\lambda$ was gradually increased until the steady state ceased to exist. Specifically, the strain was increased in steps of $\delta \lambda=0.01$; between two successive changes of $\lambda$, the simula- 
tions were run for 1000 units of time. It is seen that the results of these simulations and the theoretical calculations are in an excellent agreement. Figure 4(b) also shows the linear stability of a homogeneous BZ gel described by the following equation:

$$
1-2 u^{*}\left[1+f q\left(u^{*}+q\right)^{-2}\right]=\varepsilon\left(1-\phi_{0} \lambda^{-1} \lambda_{\perp}^{-2}\right)^{-1},
$$

where $u^{*}$ is given by Eq. (23). Equation (36) is obtained by solving the eigenproblem in Eqs. (33) and (34) for spatially uniform concentrations $\left[\widetilde{u}^{\prime \prime}=0\right.$ in Eqs. (33) $]$, and requiring that $\operatorname{Re} \Lambda=0$.

It is evident from Fig. 4(b) that changing the lateral stretching $\lambda$ at a given stoichiometric parameter $f$ can result in a switching between the oscillatory and nonoscillatory regimes of the $\mathrm{BZ}$ reaction. The lateral size at which the switching between the reaction regimes occurs depends on the value of $f$, and on the length $L_{p}$ of the BZ catalystcontaining region. It is also seen in Fig. 4(b) that the domain of the oscillatory regime decreases with decreasing patch length $L_{p}$. In other words, gel samples having short BZ patches must be stretched to greater tensile strains in order to induce the $\mathrm{BZ}$ oscillations.

It is worth noting that the initial unstretched length of swollen polymer gels depends on the cross-link density, $c_{0}$. At a given temperature, the degree of swelling decreases with an increase in the cross-link density. The latter fact is demonstrated in Fig. 4(b) by the thin, dashed horizontal lines, which show the equilibrium swelling in the gel at three values of the cross-link densities. These degrees of swelling were obtained as described in Refs. [6,12], and correspond to a temperature of about $20{ }^{\circ} \mathrm{C}$. It is evident from Fig. 4(b) that in the absence of an applied force and a given value of $f$, whether or not the heterogeneous gels exhibit chemical oscillations depends on the length of patch $L_{p}$ and the crosslink density in the gel. From Fig. 4(b), we can deduce that a densely cross-linked gel might require application of a larger external force than a loosely cross-linked sample to reach the oscillatory domain. Thus, the induction of oscillations via small changes in applied strain or swelling are predicted to be achieved most readily for lightly cross-linked gels comprising large BZ patch lengths.

It was noted above that the numerical simulations confirm the results of the linear stability analysis. Further simulations revealed the existence of hysteresis; namely, for a certain range of the stoichiometric parameter $f$, the transition between the oscillatory and nonoscillatory regimes of the BZ reaction occurs at different values of $\lambda$ depending on whether the transition is approached from within the oscillatory or steady-state domains. Figure 5 shows the presence of an area in which the oscillatory and nonoscillatory regimes coexist in the heterogeneous gels at $L_{p}=3$ and 5, and in the homogeneous 1D gel $\left(L_{p}=\infty\right)$. In this figure, the solid lines are obtained through the linear stability analysis as described above, and the data points shown by the symbols were obtained through the numerical simulations in which the strain $\lambda$ was decreased starting from the oscillatory regime. The data points were obtained by varying $\lambda$ in steps of $\delta \lambda$ $=0.01$, and are connected by the dashed line to guide the eye. The two regimes coexist in the area between the solid and

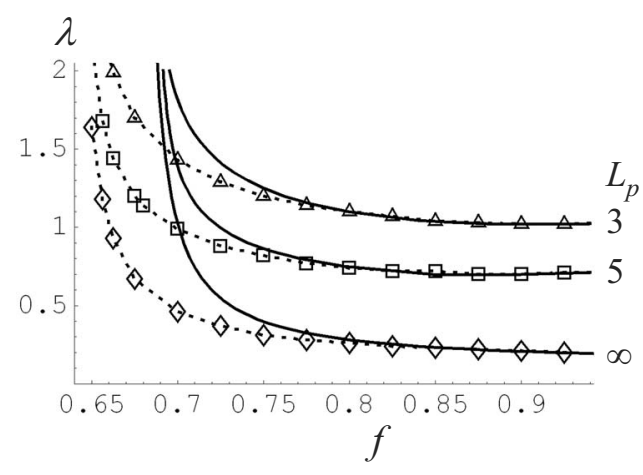

FIG. 5. Coexistence of the oscillatory and nonoscillatory regimes in the heterogeneous gel with one patch of length $L_{p}=3,5$, and $\infty$. The regimes coexist in the area between the solid and dashed curves corresponding to the same $L_{p}$. Symbols: the data obtained by the simulations.

the dashed lines. If the system is initially in the nonoscillatory regime and the dashed line is crossed from below, the system remains in the steady state until the solid line is reached. Correspondingly, if the system is initially in the oscillatory regime and the solid line is approached and crossed from above, then the oscillations continue to exist until the dashed line is crossed, and then the oscillations stop. These simulations also revealed that the area of coexistence depends on the value of the parameter $f$. As seen in Fig. 5, the coexistence area in the both heterogeneous and homogeneous 1D gels disappears as the stoichiometric parameter $f$ is increased.

The observed hysteretic effect takes place because the Oregonator exhibits the subcritical Hopf bifurcation at the values of the stoichiometric parameter of $f<1$ [15]. As shown in Ref. [15], there is a finite interval of $f$ below the bifurcation point where the phase portrait of the Oregonator [see Eqs. (7) and (8) at $u^{\prime \prime}=0$ ] consists of a stable focus and a stable large-amplitude limit cycle separated by an unstable small-amplitude limit cycle. At a given value of $\varepsilon$, the bifurcation value of $f$ and the length of the above interval depend on the gel stretching $\lambda$ through the value of $\varepsilon_{\lambda}$, Eq. (9). If $f$ is below the above interval, only a stable focus exists in the phase portrait. Above this interval (beyond the bifurcation point), the phase trajectories exhibit an unstable focus and a stable large-amplitude limit cycle, i.e., the large-amplitude oscillations are established in the system when the steady state loses its stability [15]. For $f$ within the above interval, the system can exhibit both the steady-state and largeamplitude oscillations depending upon the initial conditions. The latter bistability causes the hysteretic behavior with variations in $f$ and the gel stretching $\lambda$. The numerical simulations were essential in detecting the hysteretic effect shown in Fig. 5 because the linear stability analysis can only locate the bifurcation point. It is worth noting that the hysteretic effect is absent at $f>1$ as the Oregonator exhibits the supercritical Hopf bifurcation for this region [15] so the oscillatory and steady states do not coexist. We also note that at $f$ $>1$, the steady state loses its stability to the small amplitude oscillations [15].

Finally, the numerical simulations showed that the frequency of chemical oscillations within the heterogeneous BZ 


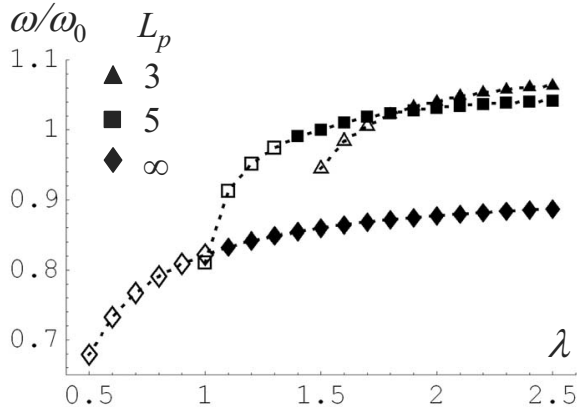

FIG. 6. Frequency of oscillation in the heterogeneous BZ gel having one patch as a function of gel stretch 1 at the patch length of $L_{p}=3,5$, and $\infty$, and $f=0.7$. The data were obtained by the simulations in the oscillatory regime (solid symbols) and in the area of coexistence of the oscillatory and nonoscillatory regimes (open symbols). $\omega_{0} \approx 0.170$ is the oscillation frequency at $L_{p}=5$ and $\lambda$ $=1.5$.

gels depends on the patch length $L_{p}$ and changes upon deformation of the sample. Figure 6 presents the oscillation frequency, $\omega$, as a function of the stretch $\lambda$ for the gel having one patch of length $L_{p}=3,5$, and $\infty$ at $f=0.7$. The frequency is normalized to the value of $\omega_{0} \approx 0.170$, which is the frequency of oscillations at $L_{p}=5$ and $\lambda=1.5$. (We note that the latter value was chosen for convenience; other choices could also have been used.) In Fig. 6, the open symbols mark the data obtained at $\lambda$ within the area of coexistence of the oscillatory and nonoscillatory regimes, and the solid symbols correspond to the oscillatory regime. The data points are connected by dotted lines as a guide for the eye.

It is seen in Fig. 6 that in the oscillatory state (solid symbols), the frequency of oscillations decreases with an increase in the patch length $L_{p}$, and stretching the gel causes a slight increase in the frequency of oscillations on the order of several percent. The effect of deformations was found to be stronger in the coexistence area (open symbols in Fig. 6), to which oscillations within the gel could be driven by compressive strain. For example, at $L_{p}=5$, the oscillation frequency exhibits a drop of about $20 \%$ upon a decrease in $\lambda$ before the oscillations stop (see open squares in Fig. 6). It is worth noting that the data for $L_{p}=\infty$ (the homogeneous BZ gel of infinite size) are presented in Fig. 6 to illustrate the general trend in the dependence of $\omega$ on $L_{p}$. In practice, the global chemical oscillations might not exist in sufficiently large BZ gels, and instead, traveling chemical waves would be observed [4]. In particular, chemical waves are experimentally observed in spherical, nonresponsive gels BZ that are larger in diameter than $\sim 500 \mu \mathrm{m}$ [10]. Since the unit of length in our simulations is $L_{0} \sim 40 \mu \mathrm{m}$, traveling chemical waves are anticipated in the heterogeneous BZ gels at $L_{p}>10 L_{0}$.

\section{B. Heterogeneous gel having two $\mathrm{BZ}$ patches}

The BZ reaction processes within the two catalystcontaining patches, which are placed next to each other, are correlated through the concentration field $u$ in the gap between the patches. If the gap is sufficiently wide, the patches

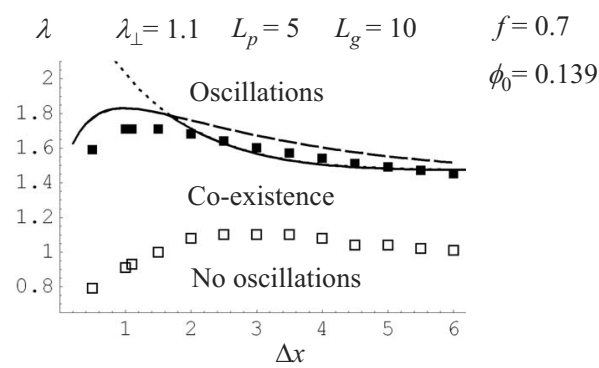

FIG. 7. Coexistence of the oscillatory and nonoscillatory regimes in the heterogeneous gel with two patches as obtained by the numerical simulations (symbols). The results of the linear stability analysis of the steady state are shown for comparison by the solid line. Also shown are the lines of linear stability with respect to the even (dashed line) and odd (short-dashed line) perturbations.

behave as independent chemical oscillators; in such cases, the effect of strain on the heterogeneous gel is similar to that described above. Varying the interpatch distance $\Delta x$ changes the strength of interaction between the BZ patches. We studied the effect of the longitudinal stretch $\lambda$ on a heterogeneous gel having two patches of length $L_{p}=5$ at the stoichiometric parameter of $f=0.7$. The regions of the oscillatory and nonoscillatory regimes in this system are mapped on the $\Delta x-\lambda$ plot shown in Fig. 7 . In the plot, the solid curve represents the position of the Hopf bifurcation point obtained by the linear stability analysis, and the symbols show the results obtained via the numerical simulations of the dynamics of the system. The procedure to obtain the data points was the same as described in the previous section, i.e., the stretch $\lambda$ was increased (or decreased) in a stepwise manner until the steady-state (or oscillatory) regime ceased to exist. The simulations were run for 2000 units of time at each value of $\lambda$. The solid symbols mark the upper boundary of the steadystate domain, and the open symbols correspond to the lower boundary of the oscillatory regime's domain; both boundaries were located by varying $\lambda$ in increments of $\delta \lambda=0.01$. As indicated in Fig. 7 , the steady-state and oscillatory regimes coexist in the area between the lines formed by the open and solid symbols. Figure 7 also shows that the results of the linear stability analysis (solid line) and numerical simulations (solid symbols) are in a fairly good agreement.

Figure 7 demonstrates that at $\Delta x>1$, a decrease in the interpatch distance (which causes the patches to interact more strongly) enlarges the steady-state domain. This effect was previously observed in $2 \mathrm{D}$ simulations of heterogeneous, nonresponsive BZ gels when $\Delta x$ was decreased at constant $\lambda[8]$. Figure 7 also reveals that at $\Delta x<1$, the size of the steady states domain diminishes. The latter behavior occurs because at $\Delta x \rightarrow 0$, the two individual patches form a single BZ patch of length $2 L_{p}$, and longer patches exhibit a larger oscillatory domain than shorter ones, as shown in the previous section. Finally, the plot also indicates that for $\Delta x$ $\leq 3$, the area of coexistence of the oscillatory and steadystate regimes increases with a decreasing interpatch distance $\Delta x$.

The numerical simulations revealed that in the oscillatory regime, the chemical oscillations within the two patches proceed with a constant phase difference equal to either 0 or to 


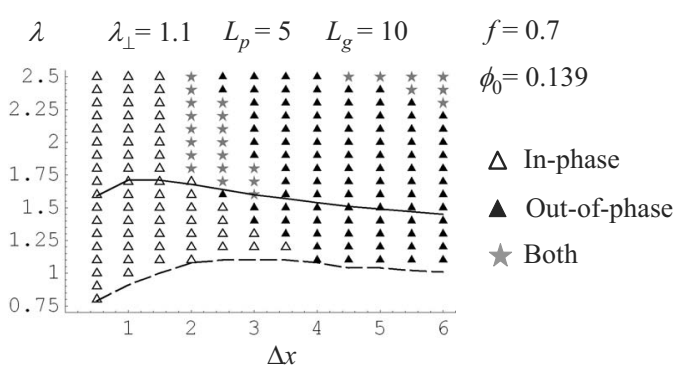

FIG. 8. Domains of the in-phase and out-of phase modes of synchronization in the heterogeneous gel with two patches as obtained by the numerical simulations. Also shown are the upper and lower stability boundaries of the steady state (see Fig. 6).

$\pi$ (in-phase or out-of-phase synchronization, respectively). The observed mode of synchronization was found to depend upon the interpatch distance, $\Delta x$, and the sample length in the deformed state, $\lambda$. Furthermore, the simulations showed that the synchronization modes coexist at some $\Delta x$ and $\lambda$, with the mode selection being dependent upon the initial conditions and the history of deformation.

Figure 8 presents the result of mapping the domains of the in-phase and out-of-phase modes of oscillation in the $\Delta x-\lambda$ coordinates. In Fig. 8, the open and solid triangles indicate the respective in- and out-of-phase oscillatory regimes, and the stars show the points where both of these synchronization modes are observed. For convenience, Fig. 8 also shows the upper and lower stability boundaries of the steady state (see Fig. 7). To obtain the map in Fig. 8, we initiated the simulations at the values of $\lambda$ that correspond to the steady ( $\lambda$ $=0.5)$ or the oscillatory $(\lambda=2.5)$ states, and then respectively increased or decreased $\lambda$ in a stepwise manner, with a step size of 0.1 . For each point in the oscillatory regime, we considered both the in- or out-of-phase oscillations as the initial conditions and then observed which of these modes remained stable (or if both were stable). The simulations showed that if a transition between the two modes of oscillation is possible, the transition might take as long as 2000 units of time. To ensure that we captured such transition points, the simulations were run for 5000 time steps at each value of $\lambda$.

As shown in Fig. 8, closely spaced patches with $\Delta x$ $\leq 1.5$ oscillate in phase at all the considered values of the applied stretch $\lambda$. The out-of-phase oscillations prevail over the in-phase mode if the patches are placed at a distance of $\Delta x \geq 3.5$. The simulations also revealed that the longitudinal strain could cause a transition between the in-phase and outof-phase modes at an interpatch distance in the intermediate range of $1.5<\Delta x<3.5$, and if the patches are located sufficiently far from each other at $\Delta x \geq 5.5$ (see Fig. 8).

It is important to note that the dominance of one or another synchronization mode near the Hopf bifurcation point can be determined through the linear stability analysis under the proper boundary conditions. It is reasonable to assume that symmetric (even) and antisymmetric (odd) perturbations of the steady state will give rise to the respective in-phase and out-of-phase oscillations. The stability of the steady state with respect to these perturbations can be determined by obtaining the eigenvalues corresponding to the even and odd eigenfunctions of Eqs. (33)-(35). Equations (33)-(35) admit both the even and odd eigenfunctions because the equations contain only the second spatial derivatives and the steady state is symmetric due to the chosen boundary conditions and the spatial symmetry of the system (see Fig. 2). At the origin, the even and odd eigenfunctions have the property of $\widetilde{u}^{\prime}(0)$ $=0$ and $\widetilde{u}(0)=0$, respectively. Therefore, the corresponding eigensolutions could be obtained by solving Eqs. (33)-(35) for half of the system at $x \geq 0$ with the above properties imposed as boundary conditions at $x=0$.

The results of the stability analysis of the even and odd perturbations are presented in Fig. 7 by the dashed and shortdashed lines, respectively. As seen in Fig. 7, the solid line, which was obtained through the stability analysis of the whole system with no a priori assumption on the symmetry of perturbation, coincides with the dashed line at $\Delta x<1.7$, and with the short-dashed line at $\Delta x>1.7$. This observation means that at a short interpatch distance, the steady state loses its stability against the even or symmetric perturbations, so that the in-phase oscillations should develop in the system. In contrast, if the patches are placed sufficiently far from each other, the odd perturbations become unstable first, and this should result in the out-of-phase mode of oscillation. Comparison of Figs. 7 and 8 shows that the results of the linear stability analysis and the numerical simulations are in agreement with respect to the mode selection in the vicinity of the Hopf bifurcation point.

Further analysis of the data presented in Fig. 8 revealed that the in-phase and out-of-phase oscillations exhibit different behavior upon stretching the gel sample having two BZ patches. This result is demonstrated in Figs. 9(a)-9(d), which show frequency of the synchronized oscillations as a function of the stretch $\lambda$ at the interpatch distance of $\Delta x=1,2,4$, and 6, respectively. In Fig. 9, the oscillation frequency in the system of two patches is shown relative to the frequency $\omega_{0} \cong 0.170$ in a single patch at $L_{p}=5$ and $\lambda=1.5$. The solid triangles mark the data points obtained in the course of stretching the sample starting from the nonoscillatory state at $\lambda=0.5$. The open squares and diamonds correspond to the data obtained during the contraction of the sample starting from the respective symmetric (in-phase) and asymmetric (out-of-phase) initial conditions within the oscillatory domain at $\lambda=2.5$.

Figure 8 indicates that at $\Delta x=1$, only the in-phase oscillations take place in the considered range of $\lambda$, and that only the out-of-phase mode exists at $\Delta x=4$. It is seen in Figs. 9(a) and 9(c) that frequency of oscillation as a function of the sample length is quite different for the in-phase and out-ofphase modes. Namely, frequency of the in-phase oscillations monotonously increases as the sample is stretched at $\Delta x=1$ [Fig. 9(a)], whereas at $\Delta x=4$, frequency of the out-of-phase mode exhibits a maximum and then decreases with an increase in the sample length [Fig. 9(c)]. Next, Fig. 8 shows that at $\Delta x=2$ and 6 , the in-phase and out-of-phase modes coexist at sufficiently high values of $\lambda$. Owing to this coexistence, frequency as a function of the sample length exhibits two branches at high values of $\lambda$ as seen in Figs. 9(b) and 9(d). At $\Delta x=2$, the in-phase oscillations take place throughout the whole range of $\lambda$ considered, whereas the out-ofphase oscillations are found only in the elongated state under 
$\Delta$ Extension Contraction: $\square$ start in-phase, $\diamond$ start out-of-phase
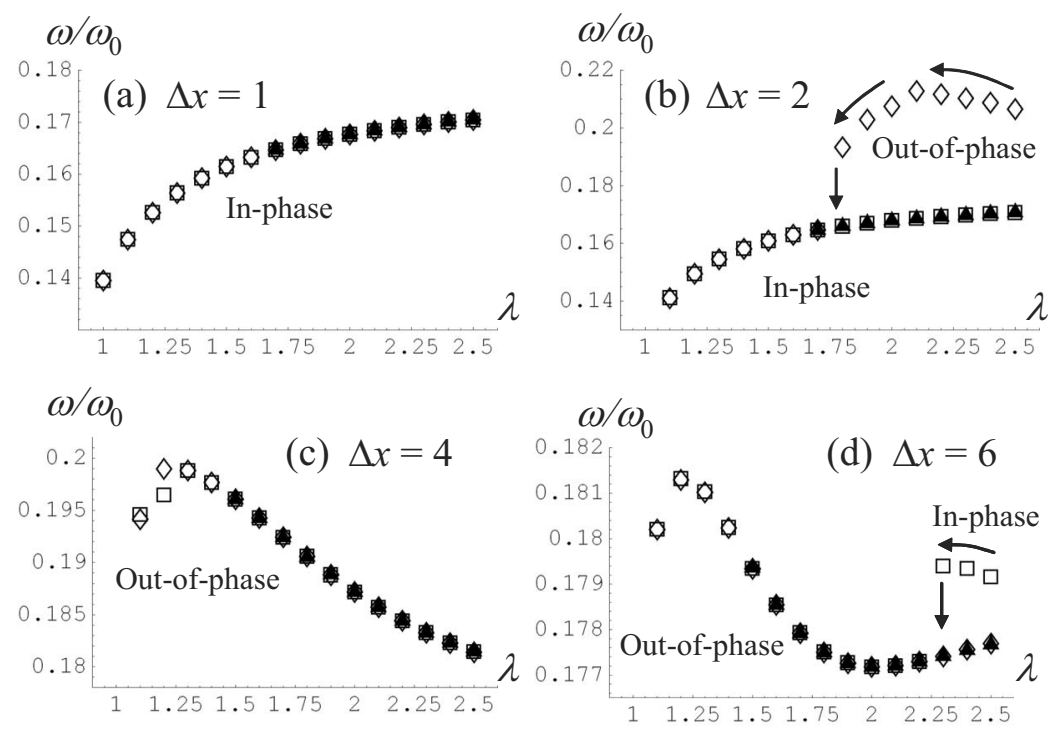

FIG. 9. Frequency of synchronized oscillations in the heterogeneous BZ gel having two patches as a function of sample length in the elongated state at various interpatch distances. $\omega_{0} \approx 0.170$ is the oscillation frequency in a single patch at $\lambda=1.5$.

appropriate initial conditions [Fig. 9(b)]. In contrast, at $\Delta x$ $=6$, the out-of-phase mode dominates, and the in-phase oscillations are observed only at high stretch ratios [Fig. 9(d)]. Finally, it is worth noting that although the characteristic features of the function $\omega(\lambda)$ are determined by the mode of oscillation (in- or out-of-phase), the magnitude of the effect depends on the interpatch distance $\Delta x$. Namely, variations in the frequency of oscillations upon changing $\lambda$ become less prominent as the distance between patches increases (Fig. 9).

There are two separate effects that contribute to the dependence of the chemical oscillation frequency on $\lambda$ presented in Fig. 9. One effect is due to the changes in the volume fraction of polymer and patch length in the course of stretching (or compressing) the gel sample. This effect is responsible for the variations in the frequency of oscillation in a single BZ patch shown in Fig. 6. Another effect is specific to the gel having two BZ patches and is due to the variations in strength of the interaction between the patches caused by the changes in the interpatch distance in the course of deformation. In order to extract the effect of variation in the interpatch distance, we normalized the function $\omega(\lambda)$ for the system with two patches to the function $\omega_{0}(\lambda)$ for the system with one patch at the same patch length. This normalization procedure was applied to the $\omega(\lambda)$ functions for the in-phase and out-of-phase oscillations shown in Fig. 9. Figure 10(a) shows the result of this renormalization and indicates a qualitative difference in behavior of the in-phase and out-of-phase oscillations. First, at the same value of $\lambda$, the out-of-phase mode in the system having two patches exhibits a higher frequency of oscillations than that in the system having one patch. In contrast, the frequency of the in-phase oscillations in two patches is lower than that in a single patch. Second, the frequency of the out-of-phase oscillations increases notably upon compression (i.e., with a decrease in $\lambda$ ), whereas the in-phase mode exhibits a slight increase in the frequency of oscillation upon extension.

It is seen in Fig. 10(a) that the effect of $\lambda$ on the oscillation frequency is stronger if the patches are placed close to each other, cf. the out-of-phase mode at $\Delta x=4$ and 6 . The dependence of the frequency of oscillations on the interpatch distance is further illustrated in Fig. 10(b), which presents the normalized frequency as a function of $\Delta x$ at the values of $\lambda$ of 1.5 and 2.2. Figure 10(b) shows that the in-phase oscillations exist only at small $\Delta x(\Delta x \leq 2.5$ in the figure $)$, and that their frequency is lower that the one in a single patch and changes very little under variations of $\Delta x$. In contrast, the out-of-phase oscillations exist only at sufficiently high $\Delta x$ (see also Fig. 8), their frequency is greater than in the system having one patch, and the latter difference diminishes with an increase in the interpatch distance [Fig. 10(b)]. It is
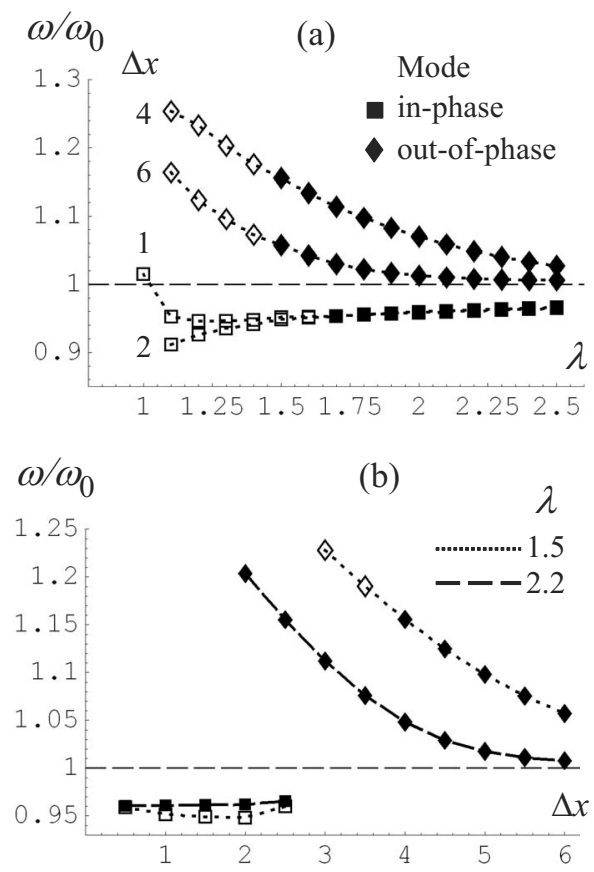

FIG. 10. Normalized frequency of the in-phase and out-of-phase oscillations as a function of (a) gel stretch $\lambda$ and (b) interpatch distance $\Delta x . \omega_{0}$ is the $\lambda$-dependent oscillation frequency in a single patch at $L_{p}=5$ shown in Fig. 6 . Solid symbols: oscillatory regime. Open symbols: oscillatory and steady states coexist. 


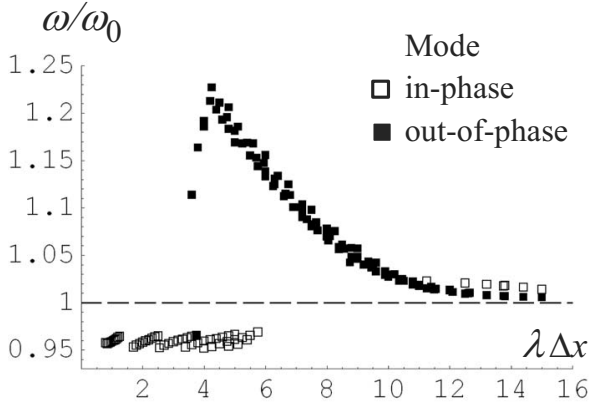

FIG. 11. Normalized frequency of the in-phase (open symbols) and out-of-phase (solid symbols) oscillations as a function of the actual interpatch distance $\lambda \Delta x$. Shown data points correspond to the oscillatory regime in Fig. 8. $\omega_{0}$ is the $\lambda$-dependent oscillation frequency in a single patch at $L_{p}=5$ shown in Fig. 6 .

notable in Fig. 10(b) that the effect of $\Delta x$ on the frequency is weaker in the sample with a greater elongation (cf. the outof-phase mode at $\lambda=1.5$ and 2.2).

As shown in Figs. 10(a) and 10(b), the behavior of the normalized frequency of oscillation in the system having two patches under variations in the elongation and interpatch distance, respectively, are similar. In addition, Fig. 10 shows that an increase in the actual distance between patches, $\lambda \Delta x$ (achieved either by changing either $\lambda$ or $\Delta x$ ), weakens the interaction between the two patches, and the latter behavior is reflected in the convergence of the values of $\omega$ and $\omega_{0}$ (see Fig. 10). In fact, the actual interpatch distance $\lambda \Delta x$ could be considered as a control parameter. To demonstrate the latter statement, in Fig. 11 we show the normalized frequency for all of the data points within the oscillatory domain in Fig. 8 as a function of $\lambda \Delta x$. It is seen that the frequencies corresponding to the in-phase and out-of-phase oscillations form distinct branches in the diagram. The characteristic features of the two branches combine all of the features discussed above concerning the two modes of oscillation. In particular, the in-phase mode exhibits a weak dependence of the frequency on the actual interpatch distance, and exists only at sufficiently small $(\lambda \Delta x<6)$ or great $(\lambda \Delta x>12)$ interpatch spacings. Additionally, the out-of-phase mode exists at sufficiently high distances $(\lambda \Delta x>3)$, and its frequency is a notable decreasing function of $\lambda \Delta x$. Finally, the two modes of oscillation coexist at some interpatch distances (see Fig. 11).

\section{CONCLUSIONS}

We undertook theoretical and computational studies to determine the effect of mechanical deformations on the behavior of heterogeneous gels undergoing the oscillatory BZ reaction. We considered a $1 \mathrm{D}$ model of the heterogeneous BZ gel, in which the catalyst is located within specific patches. The BZ reaction was assumed to have no effect on the swelling of the gel (i.e., the gel is nonresponsive). The system was modeled by using a modified Oregonator model that took into account the presence of polymer. Within this model, the deformations were shown to affect the system's behavior only through the effective diffusion constant and the parameter $\varepsilon$ of the Oregonator model appropriate for BZ reactions within a gel. In particular, these parameters now depend on the deformation, parametrized by the lateral stretch $\lambda$, and on the volume fraction of polymer in the deformed sample.

We demonstrated that the steady-state solutions of the model could be found by solving a system of nonlinear algebraic equations. We applied this approach to obtain the steady states for gels having one and two BZ patches, and then studied the stability of the solutions through the linear stability analysis. The stability analysis revealed that the longitudinal deformation could induce a transition between the oscillatory and nonoscillatory states of the system. In heterogeneous gels with a single BZ patch, the deformation at the transition point was found to depend on stoichiometry of the $\mathrm{BZ}$ reaction (the parameter $f$ in the Oregonator model) and on the patch length. In a gel having two patches, the critical deformation depended also on the interpatch distance.

The numerical simulation of the dynamics of the heterogeneous BZ gels revealed the existence of hysteresis in the system at $f<1$. Namely, the transition between the oscillatory and steady-state regimes occurs at different longitudinal deformations depending upon whether the transition is approached from the within the oscillatory or steady-state domains. The range of longitudinal strains where the two regimes coexist in the gel having one patch was shown to depend on the patch length and the parameter $f$. For gels encompassing two patches, the interpatch distance was found to affect the coexistence area.

Two modes of the BZ oscillations were observed in the numerical simulations of the gels having two patches, namely, the in-phase and the out-of-phase modes. The two modes of oscillation were shown to exhibit a qualitatively different behavior of the oscillation frequency as a function of the actual distance, $\lambda \Delta x$. The frequency of the in-phase oscillations is lower than that in the system with a single patch and exhibits a weak dependence on the interpatch distance and the elongation. In contrast, the out-of-phase oscillation has a higher frequency than within a single patch, and the magnitude of the effect increases notably as the patches become close to each other. The in-phase oscillations take place if the patches are placed sufficiently close to each other, whereas the out-of-phase mode dominates at a greater interpatch distances. The linear stability analysis was demonstrated to provide a correct prediction of the mode selection close to the Hopf bifurcation point. The longitudinal deformations were shown to be capable of switching between the two modes depending upon the interpatch distance. We demonstrated that the actual distance between the patches $\lambda \Delta x$ could be considered as the control parameter.

In future studies, we will extend the above model to the case of chemo-responsive BZ gels, and will analyze the effects of mechanical deformation on the dynamical patterns in these systems. The ability to controllably alter the dynamic behavior of BZ gels through mechanical deformations opens up the possibility of using these materials to design chemomechanical sensors. With the recent development of the $3 \mathrm{D}$ gLSM [7], we can now probe the extent to which such heterogeneous BZ gels can sense not only a tensile or compressive strain over the whole sample, but also other forms of mechanical deformations, such as a localized impact or pressure. Further, we anticipate that this tractable treatment of 
strain-altered oxidative reactions within spatially heterogeneous gels will serve as a physical model to predict effects of initial spacing, domain size, and local strain on induction of biocatalytic reactions, such as metal-activated enzyme activation within biological hydrogels and cell membranes.

\section{ACKNOWLEDGMENTS}

Funding from USARO (to V.V.Y. and A.C.B.) and from the MIT NSF MRSEC (to K.J.V.V.) is gratefully acknowledged.
[1] M. K. Beyer and H. Clausen-Schaumann, Chem. Rev. 105, 2921 (2005).

[2] O. Kuksenok, V. V. Yashin, and A. C. Balazs, Soft Matter 3, 1138 (2007).

[3] R. Yoshida, T. Takahashi, T. Yamaguchi, and H. Ichijo, J. Am. Chem. Soc. 118, 5134 (1996).

[4] R. Yoshida, Bull. Chem. Soc. Jpn. 81, 676 (2008).

[5] V. V. Yashin and A. C. Balazs, Science 314, 798 (2006).

[6] V. V. Yashin and A. C. Balazs, J. Chem. Phys. 126, 124707 (2007).

[7] O. Kuksenok, V. V. Yashin, and A. C. Balazs, Phys. Rev. E 78, 041406 (2008).

[8] V. V. Yashin and A. C. Balazs, Phys. Rev. E 77, 046210 (2008).
[9] T. Yamaguchi, L. Kuhnert, Zs. Nagy-Unvarai, S. C. Muller, and B. Hess, J. Phys. Chem. 95, 5831 (1991).

[10] R. Aihara and K. Yoshikawa, J. Phys. Chem. A 105, 8445 (2001).

[11] J. J. Tyson and P. C. Fife, J. Chem. Phys. 73, 2224 (1980).

[12] V. V. Yashin and A. C. Balazs, Macromolecules 39, 2024 (2006).

[13] O. Steinbock, P. Kettunen, and K. Showalter, Science 269, 1857 (1995).

[14] P. N. Brown, G. D. Byrne, and A. C. Hindmarsh, SIAM (Soc. Ind. Appl. Math.) J. Sci. Stat. Comput. 10, 1038 (1989).

[15] M. Mazzotti, M. Morbidelli, and G. Serravalle, J. Phys. Chem. 99, 4501 (1995). 